\section{Buchrezension zu: Evidenzbasierte Selbstmedikation}

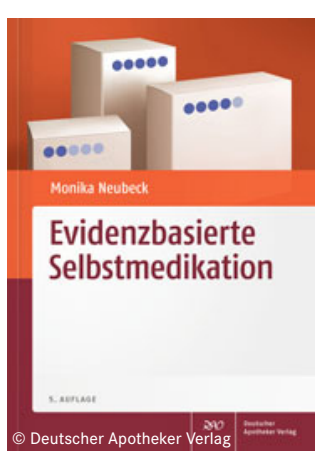

Evidenzbasierte

Selbstmedikation

Monika Neubeck

429 S., Deutscher Apotheker Verlag, 5. Aufl., 2021. HC, 39,80 €. ISBN: 9783769275568

Auch als E-Book erhältlich

DOI: $10.1007 / \mathrm{s} 12268-022-1700-2$

(C) Der Autor 2022

Die Selbstmedikation von Erkrankungen und Befindlichkeitsstörungen durch freiverkäufliche Arzneimittel stellt ein riesiges Geschäftsfeld dar. Kürzlich wurde an dieser Stelle das Buch "Selbstmedikation“ rezensiert (BIOspektrum 27: 569 (2021)) und kritisiert, dass dieses Buch leider sehr häufig den Pfad der evidenzbasierten Medizin verlässt.

Das Konkurrenzwerk dazu (deutlich umfangreicher und teurer) ist das Buch „Evidenzbasierte Selbstmedikation“. In insgesamt 42 Kapiteln werden häufige Befindlichkeitsstörungen und Erkrankungen von A wie Akne bis Z wie Zahnungsbeschwerden abgehandelt und damit ein ähnliches Spektrum wie im Vergleichsbuch erfasst. Alle Kapitel sind identisch aufgebaut: Zunächst erfolgt eine kurze Darstellung von Pathophysiologie, Klinik und übergreifenden Therapieprinzipien der Krankheitsentität. Anschließend werden die einzelnen Arzneistoffe hinsichtlich Wirkung, Anwendung, unerwünschter Wirkungen und möglicher Interaktionen besprochen. Jedes Kapitel wird durch eine Übersicht über typische Han- delspräparate und ein recht ausführliches und aktuelles Literaturverzeichnis abgeschlossen. Das Kernstück eines jeden Kapitels ist die zusammenfassende Bewertung jedes Arzneistoffs in einem 5-Punke-System von 0-5 Punkten. Es ist der Autorin hoch anzurechnen, dass sie versucht hat, für alle Arzneistoffe wissenschaftliche Literatur zusammenzutragen.

Der Knackpunkt des Buchs ist jedoch aus Sicht des Rezensenten eben diese Punktebewertung. Im Vorwort gibt die Autorin ehrlich zu, dass man evidenzbasierte Medizin auch als Verkaufsargument in der Apotheke verwenden kann, und an dieser Stelle rutscht das Buch in einen Interessenkonflikt zwischen Wissenschaft und Wirtschaft: In vielen Fällen ist die Bewertung der Arzneimittel sehr positiv, sogar viel zu positiv, offenbar mit der Intention, den Umsatz nicht zu drücken. Beispiele für eine zu wohlwollende Beurteilung sind die mit 4 Punkten bewerteten Ginkgopräparate für Demenz oder die Gabe von Acetylsalicylsäure (obsolet) bei grippalen Infekten. Sogar die bei Arthrose wirkungslosen Chondroitinpräparate erhalten 2 Punkte, obwohl 0 Punkte angemessen wären.

Insgesamt ist das Buch in seiner Aufmachung und im Preis angemessen. Es kann interessierten Lesern mit einer sehr guten Portion kritischer Distanz zur Information über wichtige Prinzipien der Selbstmedikation empfohlen werden.

Roland Seifert,

Medizinische Hochschule Hannover, seifert.roland@mh-hannover.de Diese Rezension erscheint Open Access.
* Funding note: Open Access funding enabled and organized by Projekt DEAL. Open Access: Dieser Artikel wird unter der Creative Commons Namensnennung 4.0 International Lizenz veröffentlicht, welche die Nutzung, Vervielfältigung, Bearbeitung, Verbreitung und Wiedergabe in jeglichem Medium und Format erlaubt, sofern Sie den/die ursprünglichen Autor(en) und die Quelle ordnungsgemäß nennen, einen Link zu Creative Commons Lizenz beifügen und angeben, ob Änderungen vorgenommen wurden. Die in diesem Artikel enthaltenen Bilder und sonstiges Drittmaterial unterliegen ebenfalls der genannten Creative Commons Lizenz, sofern sich aus der Abbildungslegende nichts anderes ergibt. Sofern das betreffende Material nicht unter der genannten Creative Commons Lizenz steht und die betreffende Handlung nicht nach gesetzlichen Vorschriften erlaubt ist, ist für die oben aufgeführten Weiterverwendungen des Materials die Einwilligung des jeweiligen Rechteinhabers einzuholen. Weitere Details zur Lizenz entnehmen Sie bitte der Lizenzinformation auf

http://creativecommons.org/licenses/ by $/ 4.0 /$ deed.de. 